\title{
Speed-accuracy trade-offs in computing spatial impulse responses for simulating medical ultrasound imaging
}

Jensen, Jørgen Arendt

Published in:

Journal of Computational Acoustics

Link to article, DOI:

$10.1142 / S 0218396 \times 01001248$

Publication date:

2001

Document Version

Publisher's PDF, also known as Version of record

Link back to DTU Orbit

Citation (APA):

Jensen, J. A. (2001). Speed-accuracy trade-offs in computing spatial impulse responses for simulating medical ultrasound imaging. Journal of Computational Acoustics, 9(3), 731-744.

https://doi.org/10.1142/S0218396X01001248

\section{General rights}

Copyright and moral rights for the publications made accessible in the public portal are retained by the authors and/or other copyright owners and it is a condition of accessing publications that users recognise and abide by the legal requirements associated with these rights.

- Users may download and print one copy of any publication from the public portal for the purpose of private study or research.

- You may not further distribute the material or use it for any profit-making activity or commercial gain

- You may freely distribute the URL identifying the publication in the public portal

If you believe that this document breaches copyright please contact us providing details, and we will remove access to the work immediately and investigate your claim 
Paper presented at the fourth International Conference on Theoretical and Computational Acoustics, Stazione Marittima, Trieste, Italy, May 10-14, 1999:

\title{
Speed-accuracy trade-offs in computing spatial impulse responses for simulating medical ultrasound imaging
}

\author{
Jørgen Arendt Jensen \\ Center for Fast Ultrasound Imaging, \\ Department of Information Technology, Build. 344, \\ Technical University of Denmark, \\ DK-2800 Lyngby, Denmark
}

Published in Journal of Computational Acoustics, 9(3):731744, 2001. 


\title{
SPEED-ACCURACY TRADE-OFFS IN COMPUTING SPATIAL IMPULSE RESPONSES FOR SIMULATING MEDICAL ULTRASOUND IMAGING
}

\author{
JØRGEN ARENDT JENSEN \\ Department of Information Technology, Build. 344, \\ Technical University of Denmark, DK-2800 Lyngby, Denmark \\ Received (to be inserted \\ Revised by Publisher)
}

\begin{abstract}
Medical ultrasound imaging can be simulated realistically using linear acoustics. One of the most powerful approaches is to employ spatial impulse responses. Hereby both emitted fields and pulseecho responses from point scatterers can be determined. Also any kind of dynamic focusing and apodization can be incorporated, as has been done in the Field II simulation program. Here the transducer is modeled through a set of either rectangles, triangles, or bounding lines, so that any geometry can be simulated. The response from the transducer is found by summing the spatial impulse responses from the individual elements. One of the problems in using spatial impulse responses is the abrupt changes in the responses due to the sharp transducer boundaries. Sampling the responses directly therefore have to be done at very high sampling frequencies to keep the shape and energy of the response. The high sampling frequency is unnecessary in the final signals, since the transducers used in medical ultrasound are band limited. Approaches to reduce the sampling frequency are, thus, needed to make efficient simulation programs. Field II uses time integration of the spatial impulse responses using a continuous rather than discrete time axis. This preserves the energy in the responses and makes it possible to make sub-sample interval delays for focusing. The paper discusses the consequence of the integration for the rectangular elements that uses an approximative calculation of the spatial impulse responses. Data for the accuracy as a function of sampling frequency is given, and it is shown how a sampling frequency of $100 \mathrm{MHz}$ gives similar results to using $2 \mathrm{GHz}$ sampling of the analytic solution for rectangular elements. The spatial impulse responses for the triangular and bounding line elements are found analytically, and an iterative integration routine has to be used. The Romberg integration routine is used, and the accuracy versus sampling frequency for bounding line is shown. An increased accuracy is attained for the lines compared to the rectangles, but the simulation times are significantly higher. Line elements should therefore, in this implementation, only be used very close to the transducer, and if a very high precision is needed in the calculation.
\end{abstract}

\section{Introduction}

The optimization and development of medical ultrasound systems is increasingly based on simulating the whole system. This includes the focusing and apodization during transmission of the ultrasound pulse and the dynamic receive processing performed on the multi channel data from the array transducer. Different apodization and delay profiles are employed and the design of these can be complicated. It is therefore advantageous to simulate 
the system for both point targets and for whole phantom objects with cysts and high scattering regions to evaluate both the resolution and contrast of the system.

The most frequently used approach is based on the spatial impulse response method originally developed by Tupholme ${ }^{1}$ and Stepanishen ${ }^{2},{ }^{3}$ and briefly described in Section 3. In this method the impulse response of the system is calculated for a point in space for a homogeneous medium, and hereby both the emitted and received signal for the pulsed and continuous wave case can be derived. The method relies on linear acoustics as described in Section 2, and it can handle any kind of apodization, focusing, and excitation of the transducer making it ideally suited for basic simulation of ultrasound systems.

There is, however, a number difficulties when using spatial impulse responses for simulation. The responses have dicontinuities due to the finite extend of the transducer as described in Section 4. The response will have a discontinuity, when the spherical wave from the aperture cross one of the transducer boundaries. The responses are, thus, often very broad band. Ideally the simulation should be fast and this entails using a low sampling frequency. This is in contradiction to the very broad band spatial impulse responses. The simulation should also be accurate, and this is often in contradiction to using a low sampling frequency.

The approach taken in this paper is to use the time integrated spatial impulse response, which preserves all the energy in the responses. Although the responses are broad band only the lower frequency part is used in actual simulations, since the transducer is bandlimited. The higher part of the spectrum is, thus, filtered out and it is only important to accurately model the lower frequency part. This can be done by time integrating the response. The approach, used in the Field II program mentioned in Section 5, is described for both rectangular elements that uses a far-field approximation and for triangular and bounding line elements. Finally, the accuracy and simulation times for the time integration method is described in Section 6.

\section{Linear Acoustic System}

Any linear system is fully characterized by its impulse response. From this the output signal to any input excitation can be determined and the transfer function is found by Fourier transforming the impulse response. These features has been used extensively in electrical engineering in design of filters and other circuits. The powerful concept can also be employed in linear acoustics for characterizing and optimizing acoustic systems. Here the situation is depicted in Fig. 1. An acoustic radiator is mounted in an infinite, rigid baffle and radiates into a homogeneous, non-refracting medium. The emitted pressure at a point in space is then measured with a needle hydrophone. Applying a delta function excitation on the transducer then yields the acoustical impulse response of the system. Moving the hydrophone to a new point will yield another impulse response, and it is therefore an impulse response dependent on the spatial position relative to the aperture that is measured.

Formally the emitted ultrasound pressure field $p\left(\vec{r}_{1}, t\right)$ can be described by

$$
p\left(\vec{r}_{1}, t\right)=\rho_{0} \frac{\partial v(t)}{\partial t} * h\left(\vec{r}_{1}, t\right),
$$




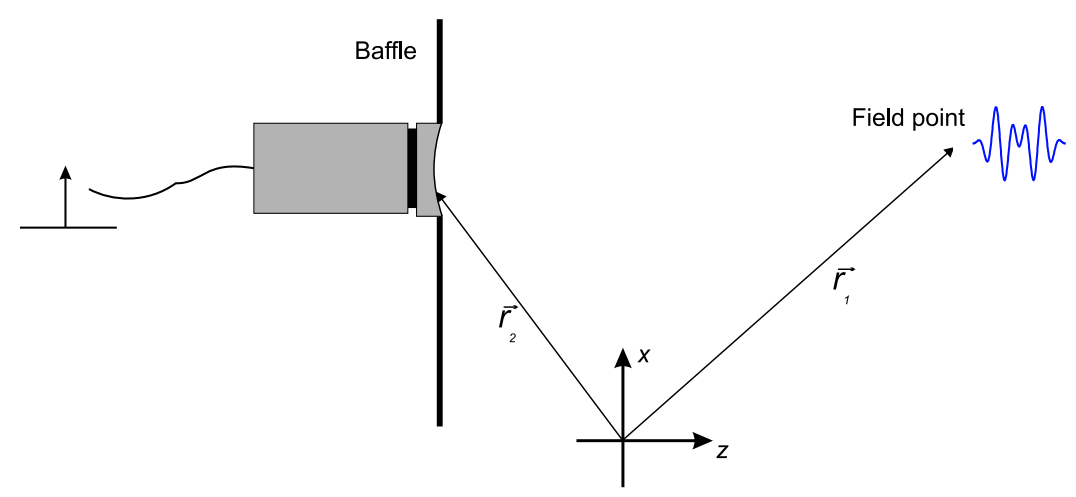

Fig. 1. Impulse response at a point in space.

where $\rho_{0}$ is the density of the medium, $v(t)$ the surface velocity of the aperture, $*$ denotes convolution and $h\left(\vec{r}_{1}, t\right)$ is the spatial impulse response of the system at location $\vec{r}_{1}$. A formal derivation of this equation can be found in the next section.

The spatial impulse response can also be used to characterize the pulse echo signal received from a small point scatterer. The received voltage signal $v_{r}\left(\vec{r}_{1}, t\right)$ is given by ${ }^{4}$ :

$$
\begin{aligned}
v_{r}\left(\vec{r}_{1}, t\right) & =v_{p e}(t) * f_{m}\left(\vec{r}_{1}\right) * \frac{\partial^{2} h_{p e}\left(\vec{r}_{1}, t\right)}{\partial t^{2}} \\
f_{m}\left(\vec{r}_{1}\right) & =\frac{\Delta \rho\left(\vec{r}_{1}\right)}{\rho_{0}}-\frac{2 \Delta c\left(\vec{r}_{1}\right)}{c} \\
h_{p e}\left(\vec{r}_{1}, t\right) & =h_{e}\left(\vec{r}_{1}, t\right) * h_{r}\left(\vec{r}_{1}, t\right)
\end{aligned}
$$

where $h_{e}\left(\vec{r}_{1}, t\right)$ is the spatial impulse response for the emitter, $h_{r}\left(\vec{r}_{1}, t\right)$ for the receiver, and $v_{p e}(t)$ is the electro-mechanical response of the transducer in both transmit and receive. The scattered signal arises from the perturbations in density $\Delta \rho\left(\vec{r}_{1}\right)$ and speed of sound $\Delta c\left(\vec{r}_{1}\right)$.

Obtaining the continuous wave fields for both the emitted and pulse echo case is straight forward since Fourier transform of the spatial impulse response gives the continuous wave response. All fields relevant in medical ultrasound can, thus, be calculated from the spatial impulse response.

\section{Derivation of spatial impulse response}

This section will give a brief derivation of the spatial impulse response in order to explain the properties and difficulties of spatial impulse response. The spatial impulse response is formally defined by

$$
h\left(\vec{r}_{1}, t\right)=\int_{S} \frac{\delta\left(t-\frac{\left|\vec{r}_{1}-\vec{r}_{2}\right|}{c}\right)}{2 \pi\left|\vec{r}_{1}-\vec{r}_{2}\right|} d S
$$

where $\left|\vec{r}_{1}-\vec{r}_{2}\right|$ is the distance to field point, $c$ is the speed of sound, and $S$ denotes the active surface of the transducer. This can be seen as a statement of Huygens' principle in 
which each point on the radiating surface emits a spherical wave. The field is then found by integrating all the spherical waves for a given time and spatial position in order to obtain the emitted pressure for one time instance and position. The situation is illustrated in Fig. 2, where the radiated spherical waves are plotted as a function of space for one given time instance. The higher amplitude spatial impulse response in front of the transducer compared to the sides is easily seen.

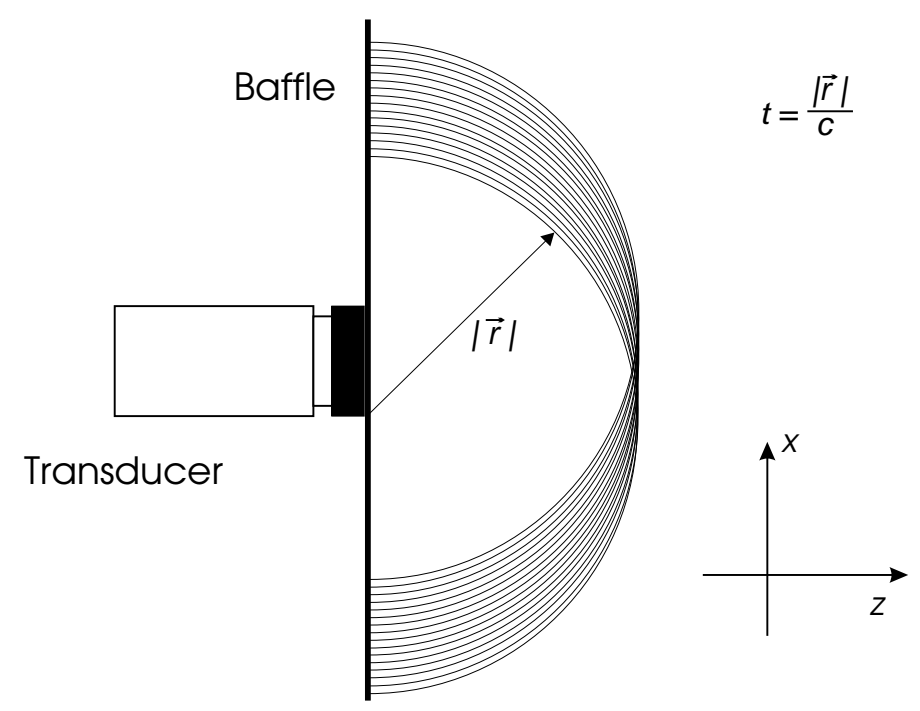

Fig. 2. Radiation of spherical waves from an aperture for a fixed time instance.

Figure 2 gives a general overview of the spatial extend of the field for a given time instance, but the responses should be evaluated for one given position as a function of time. This can be done by interchanging emitted and receiver using acoustic reciprocity, which says that "If in an unchanging environment the locations of a small source and a small receiver are interchanged, the received signal will remain the same" ${ }^{5}$. This is illustrated in Fig. 3 in which a spherical wave is emitted from the small point receiver. The spherical waves projected onto the flat plane of the aperture denoted by the gray triangular area results in circles with an origo at the field point and a radius dependent on the time as shown in Fig. 4. The spatial impulse response for a given time is then determined by which part of the circle that intersects the active aperture. This can be derived using (3.4) Rewriting into polar coordinates using

$$
\iint_{s} f(x, y) d x d y=\int_{0}^{r} \int_{0}^{2 \pi} r f(r, \theta) d \theta d r
$$

gives

$$
h\left(\vec{r}_{1}, t\right)=\int_{0}^{r} \int_{0}^{2 \pi} r \frac{\delta\left(t-\frac{|R|}{c}\right)}{2 \pi|R|} d \theta d r .
$$




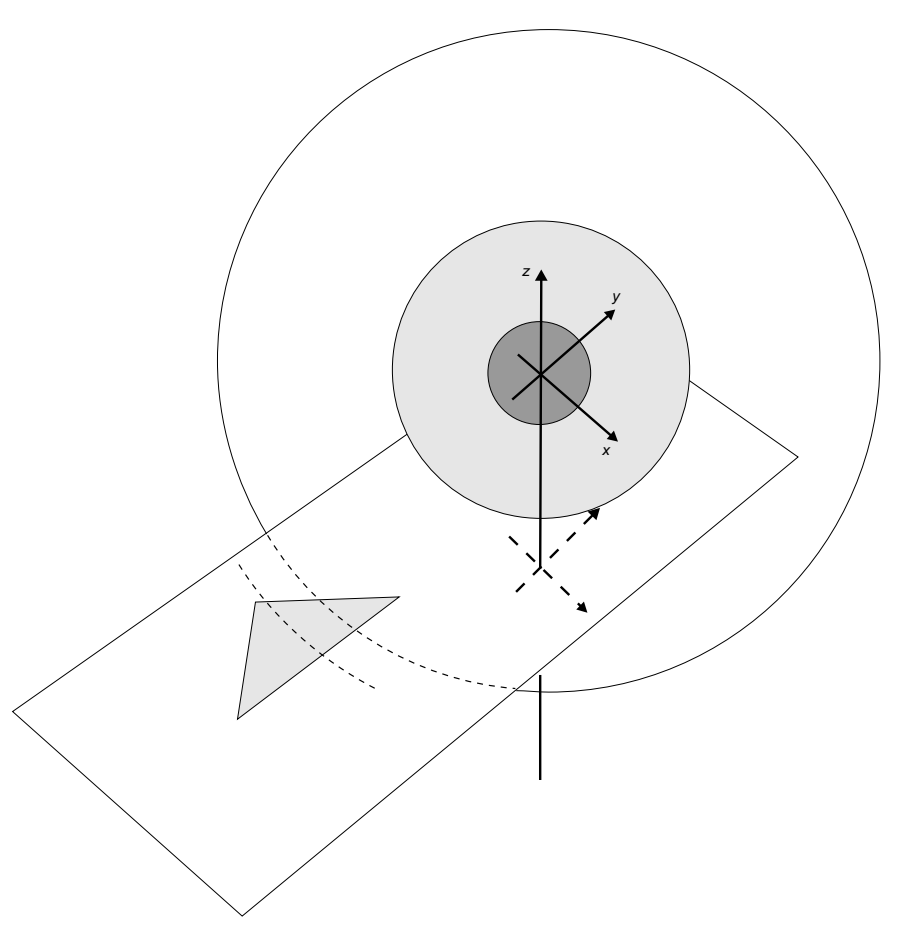

Fig. 3. Emission of spherical wave from the field point and its intersection of the aperture.

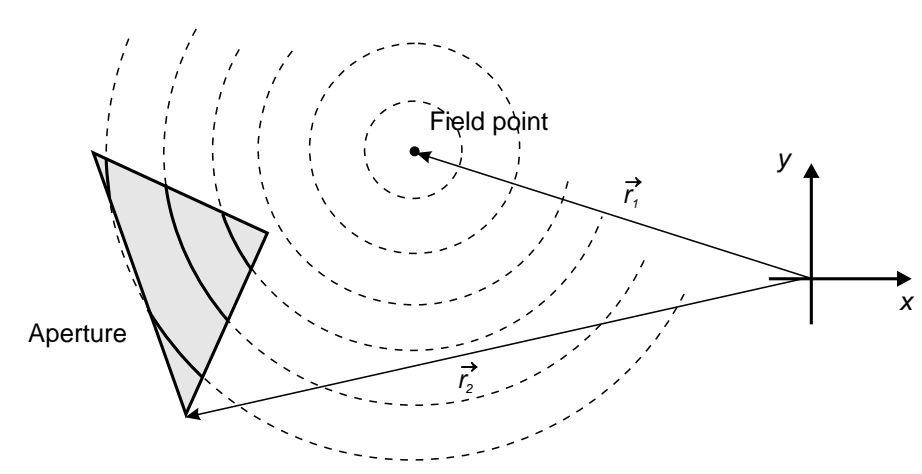

Fig. 4. Intersection of spherical waves from the field point by the aperture, when the field point is projected onto the plane of the aperture. 
The projected circles have a radius $r=\sqrt{(c t)^{2}-z^{2}}$. The distance to the field point is $R=\sqrt{z^{2}+r^{2}}$, where $z$ is the field point's height above the $x-y$ projection plane.

An example of the geometric situation for a triangular aperture is shown in Fig. 5. The field point is at one of the corner points, and the dashed line indicates the projected spherical wave for a given time instance.

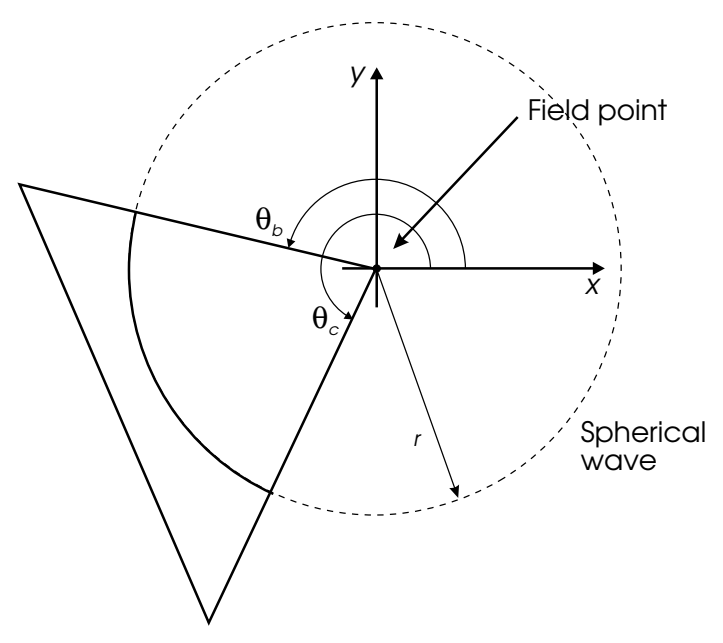

Fig. 5. Geometry for evaluating the spatial impulse response from a triangular aperture, when the field point is at one of the corner points. The dashed line indicates the projected spherical wave for a given time instance.

The first spherical wave arrives at the aperture at time $t=t_{1}=z / c$. Hereafter the fixed part of the circle between the angles $\theta_{b}$ and $\theta_{c}$ contributes to the response, and the response is:

$$
h_{T}\left(\vec{r}_{1}, t\right)=\int_{0}^{r} \int_{\theta_{b}}^{\theta_{c}} r \frac{\delta\left(t-\frac{|R|}{c}\right)}{2 \pi|R|} d \theta d r=\frac{\theta_{c}-\theta_{b}}{2 \pi} \int_{0}^{r} r \frac{\delta\left(t-\frac{|R|}{c}\right)}{|R|} d r
$$

Substituting $2 R d R=2 r d r$ gives:

$$
h_{T}\left(\vec{r}_{1}, t\right)=\frac{\theta_{c}-\theta_{b}}{2 \pi} \int_{z}^{\sqrt{z^{2}+r^{2}}} \delta\left(t-\frac{|R|}{c}\right) d R
$$

A final time substitution of $R / c=t^{\prime}$ results in

$$
h_{T}\left(\vec{r}_{1}, t\right)=\frac{\theta_{c}-\theta_{b}}{2 \pi} c \int_{t_{1}}^{t_{x}} \delta\left(t-t^{\prime}\right) d t^{\prime}=\frac{\left(\theta_{c}-\theta_{b}\right)}{2 \pi} c \quad \text { for } t_{1} \leq t \leq t_{x}
$$

Time $t_{x}$ equals the corresponding time for the point closest to origo and gives the starting point for the impulse response. $t_{1}$ is the time for the projected circle reaching the far edge of the triangle, and the response then drops off due to the smaller part of the arc intersecting the active aperture. This derivation shows, that it is the part of the arc on the aperture, that determines the magnitude of the spatial impulse response. Also the response is always 
positive. The discontinuities in the response is due to the crossing of a boundary of the aperture by the projected wave.

A simple examples of a spatial impulse response is seen in Fig. 6. The response starts
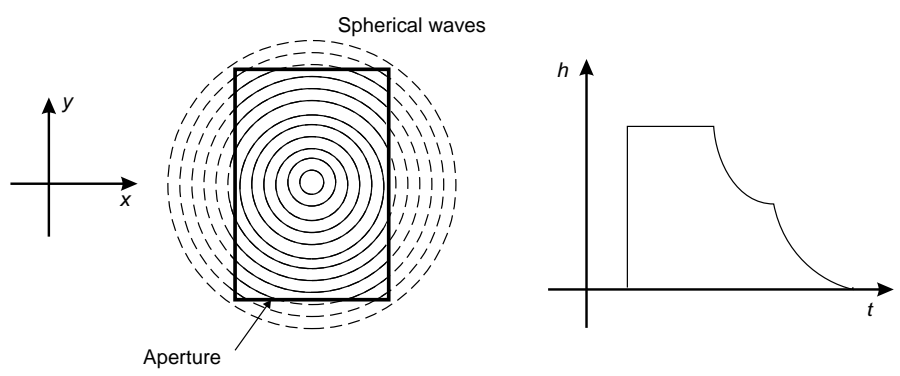

Fig. 6. Spatial impulse response from a rectangular aperture when the field point is inside the aperture.

out with a value of $c$ after the arrival of the first spherical wave at time $t=z / c$. It then drops of after crossing the first edge and the drops further, when reaching the next edges. It finally attains a value of zero, when the projected spherical waves are beyond the edges of the aperture.

An other example of spatial impulse responses is shown from a triangular in Fig. 7. The different discontinuities and variations in the responses are seen.

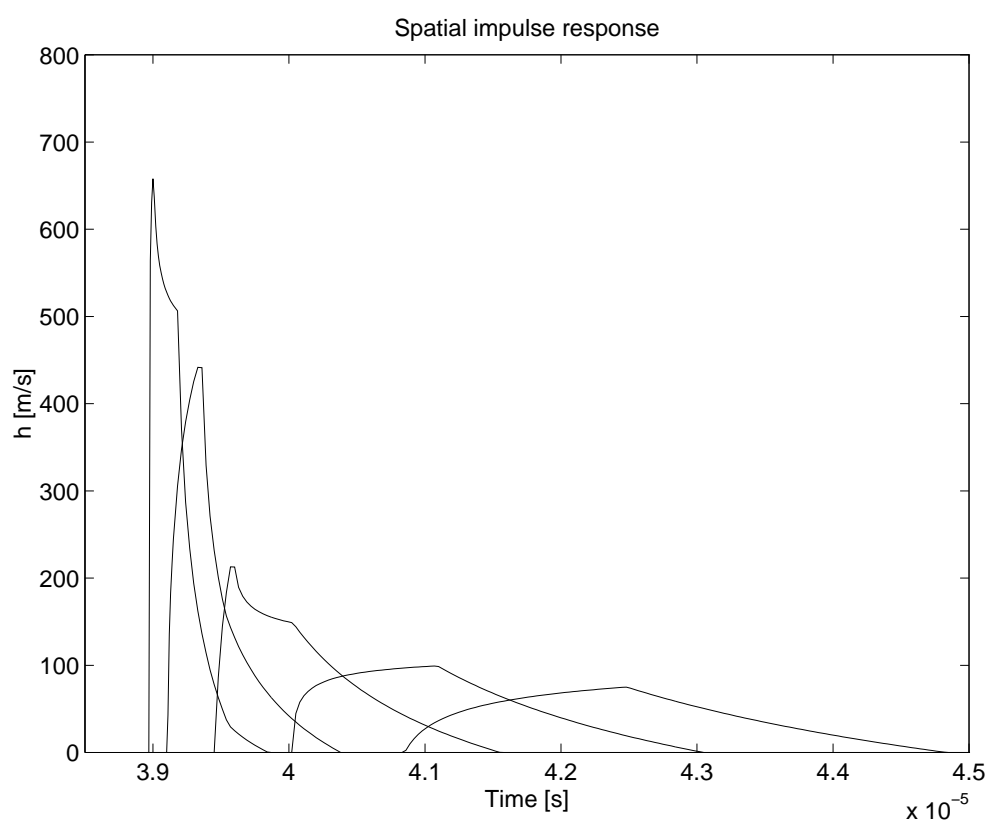

Fig. 7. Spatial impulse response from a triangular aperture for different field point positions. 


\section{Problems with spatial impulse responses}

There is a number of difficulties, when using spatial impulse responses in simulating ultrasound systems. Primarily they are difficult to calculate analytically, and equations for some commonly used transducer geometries have not been derived. This includes the elevation focused linear element and convex array transducers. Secondly, the responses are numerically difficult to handle, since they have discontinuities due to the aperture elements edges. The discontinuities generate very high frequencies in the short responses from small array transducer elements, and these are difficult to handle in a sampled simulation program.

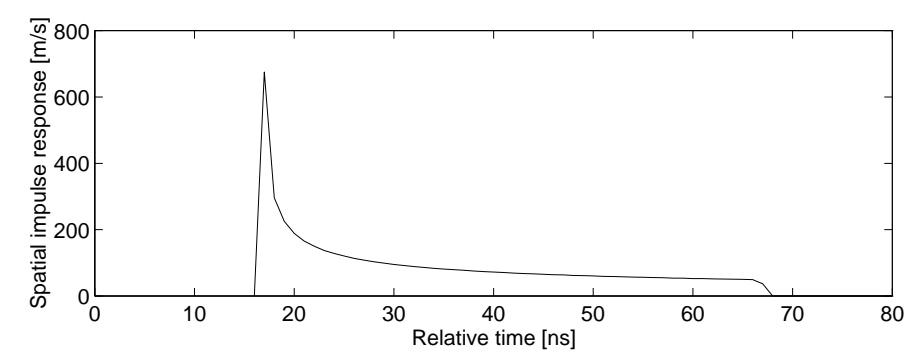

Fig. 8. Spatial impulse response from small array element.

The problems are illustrated in Fig. 8, which shows the impulse response from a small array element. The response is of very short duration and has sharp discontinuities. Such an element can be used in, e.g., a $3 \mathrm{MHz}$ array transducer and a natural choice of simulation sampling frequency would be $20 \mathrm{MHz}$. This would, however, not be sufficient for the high frequencies in the response. This is illustrated in Fig. 9, where the analytic solution for a concave transducer was evaluated using a $100 \mathrm{MHz}$ and a $2 \mathrm{GHz}$ sampling frequency. The $100 \mathrm{MHz}$ calculation distorts the response severely at the center, because the spatial impulse response here approaches a delta-function.

The solution to these problems is to divide the aperture into small elementary elements and carefully keep track of the energy of the elementary responses. Hereby any geometry transducer can be modeled as a set of elementary elements and their responses superimposed due to linearity. It should also be noted that although the spatial impulse responses are very wide bandwidth only a small part of this bandwidth is often used, since the electromechanical bandwidth of the transducer is limited. It is therefore important to keep track of all the energy in the responses and not so important to model exactly every change in the small responses, since this only has an effect at high frequencies. Ensuring that all energy is kept is here done by integrating the calculated responses on a continuous time axis and then calculate the spatial impulse response at the discrete points by taking the difference from sample point to sample point. Examples of the efficiency of this approach is shown later.

The advantages of subdizing into mathematical elements is that all transducer geometries can be handled. The phasing and thereby time delay applied on each element of the 

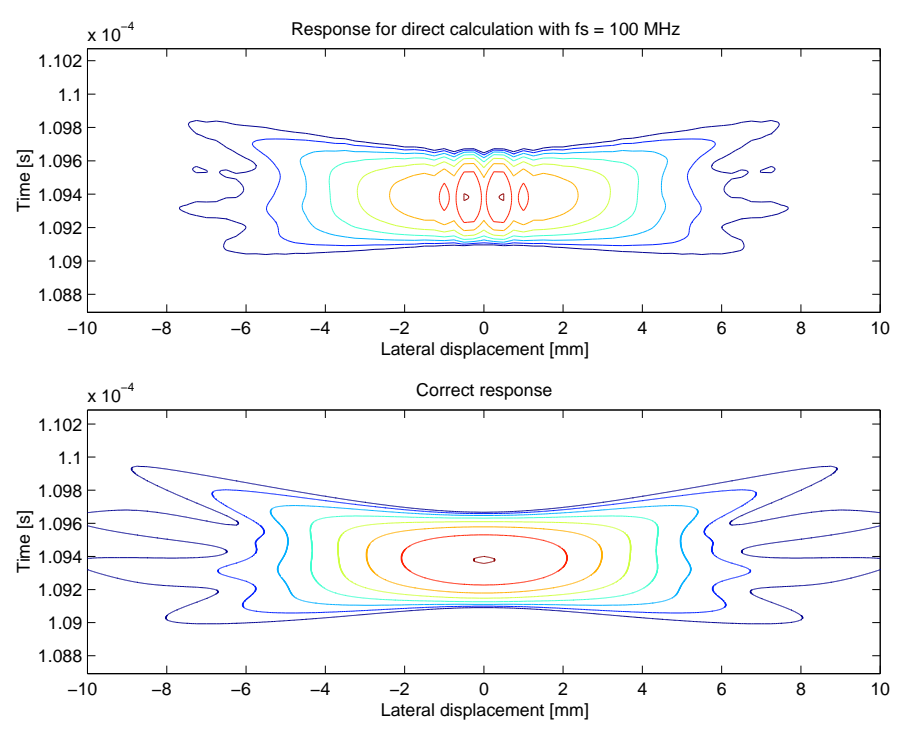

Fig. 9. Pulse-echo field from concave transducer at the focal point calculated with a sampling frequency of $100 \mathrm{MHz}$ (top) and $2 \mathrm{GHz}$ (bottom).

transducer is easily handled and the apodization, i.e. that $v(t)$ varies over the transducer surface, can also be incorporated through a simple scaling of the individual element responses. Using spatial impulse responses also ensures that all kinds of excitations of the aperture can be handled.

\section{Field II simulation program}

The division of the aperture into small mathematical elements is the method used by the Field II program. Three different types of elements are used as shown in Fig. 10. For the rectangle elements a far-field calculation of the spatial impulse response is used

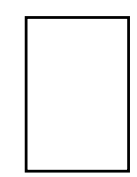

Rectangles

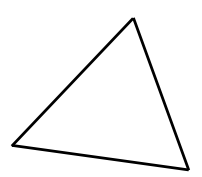

Triangles

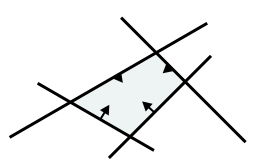

Bounding lines

Fig. 10. Basic mathematical elements used for modeling the transducer aperture in Field II.

as shown in Fig. 11. The response is very fast to calculate ${ }^{6}$ and it can be analytically integrated for giving a continuous time axis and preserving the energy in the responses. The drawback is the large number of rectangles needed when the field point is close to the aperture and when the aperture is round or oval. This can be overcome by the triangular 


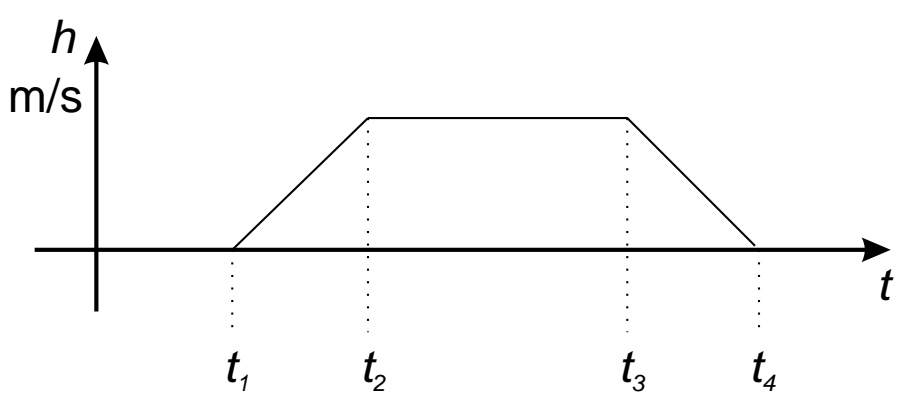

Fig. 11. Far-field response from rectangle.

element in which the full solution is used ${ }^{7}$. A Romberg integration routine ${ }^{8}$ is then used for integrating the response. The triangular element gives a better fit to complex apertures, and the calculated responses has the highest precision attainable for the give sampling frequency, when using this approach. The solution is, however, much slower than the farfield rectangular solution. The last mathematical element is constructed from bounding lines in the plane of the transducer. Hereby any shape element can be described exactly as long as it has straight edges. The method gives a very simple solution for finding the spatial impulse response ${ }^{9}$ and the Romberg method is again used for finding the integrated response.

The basic structure of the Field II program is shown in Fig. 12. The Matlab program

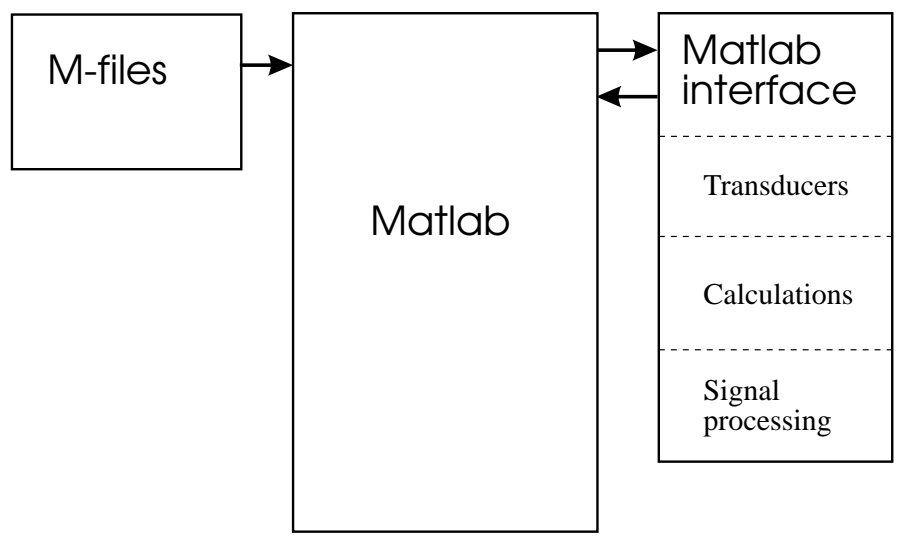

Fig. 12. Basic structure of the Field II program.

(MathWorks Inc., Massachusetts, USA) is used for interfacing with the user. This makes it possible to make scripts for performing ultrasound imaging using both for-loops and conditional statements along with all forms of signal processing and array manipulations. The actual calculations are done in the $\mathrm{C}$ program associated with the Matlab code through mex files. Only the parameters for the simulation and the resulting signals are passed 
through the Matlab code thereby making the simulation both fast and memory efficient. The basic features of the program is:

- Transducer modeled by dividing it into rectangles, triangles and bounding lines.

- C program interfaced to Matlab.

- Matlab used as front-end.

- Can handle any transducer geometry.

- Physical understanding of transducer.

- Pre-defined types: piston and concave single element, linear array, phased array, convex array, 2D matrix array.

- Any focusing, apodization, and excitation pulse.

- Multiple focusing and apodization.

- Dynamic focusing.

- Can calculate all types of fields (emitted, received, pulsed, CW)

- Can generate artificial ultrasound images (phased and linear array images with multiple receive and transmit foci).

- Data storage not necessary.

- Post-processing in Matlab 5.

- Versions for: Windows(Win 95, 98 \& NT), Linux, HP, SUN, SGI, DEC Station (ALPHA).

- Executable code is public at: http://www.it.dtu.dk/ jaj/field/

\section{Accuracy of integrated response}

The attainable accuracy of the integrated spatial impulse response approach can be seen in Fig. 13. This show the point spread function calculated at two different sampling frequencies for a 64 element linear array. The top shows the response for a sampling frequency of $100 \mathrm{MHz}$ and the bottom for $2 \mathrm{GHz}$. There is $6 \mathrm{~dB}$ between contours and the functions are essentially similar down to a level of $-48 \mathrm{~dB}$ and the only difference is that the top response is 20 times fast to calculate than the bottom response.

The same general picture is seen, when comparing a measured and a simulated response as shown in Fig. 14. A concave transducer focused at $100 \mathrm{~mm}$ with a diameter of $8 \mathrm{~mm}$ was used. A small needle was moved in front of the transducer and the scattered voltage 
12 Speed-accuracy trade-offs in simulating medical ultrasound imaging
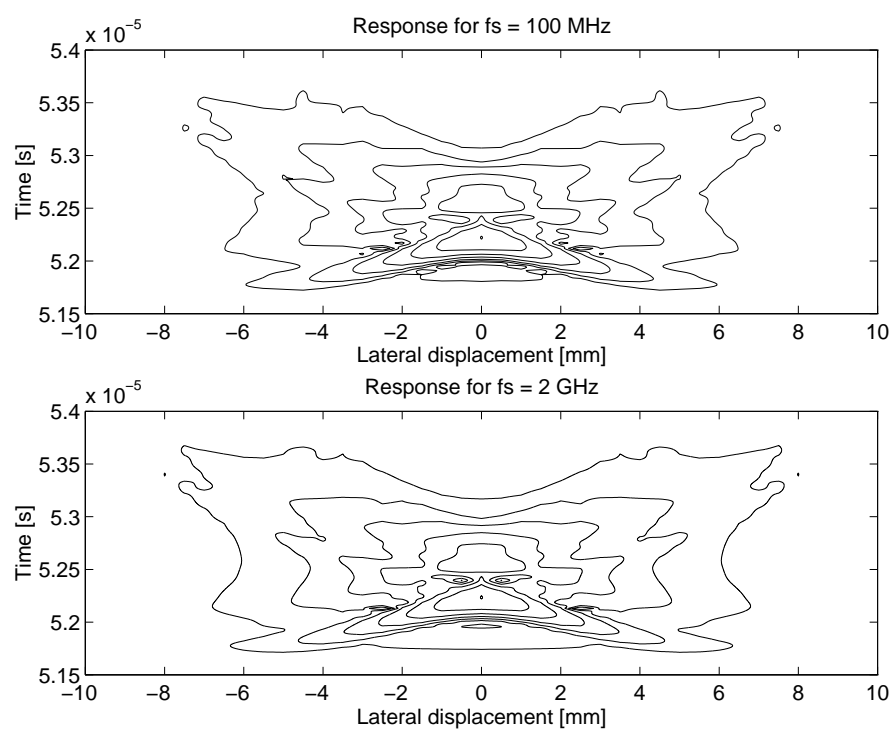

Fig. 13. Point spread function for 64 element linear array for $f_{s}=100 \mathrm{MHz}$ (top) and $f_{s}=2 \mathrm{GHz}$ (bottom) ( $6 \mathrm{~dB}$ between the contour lines).
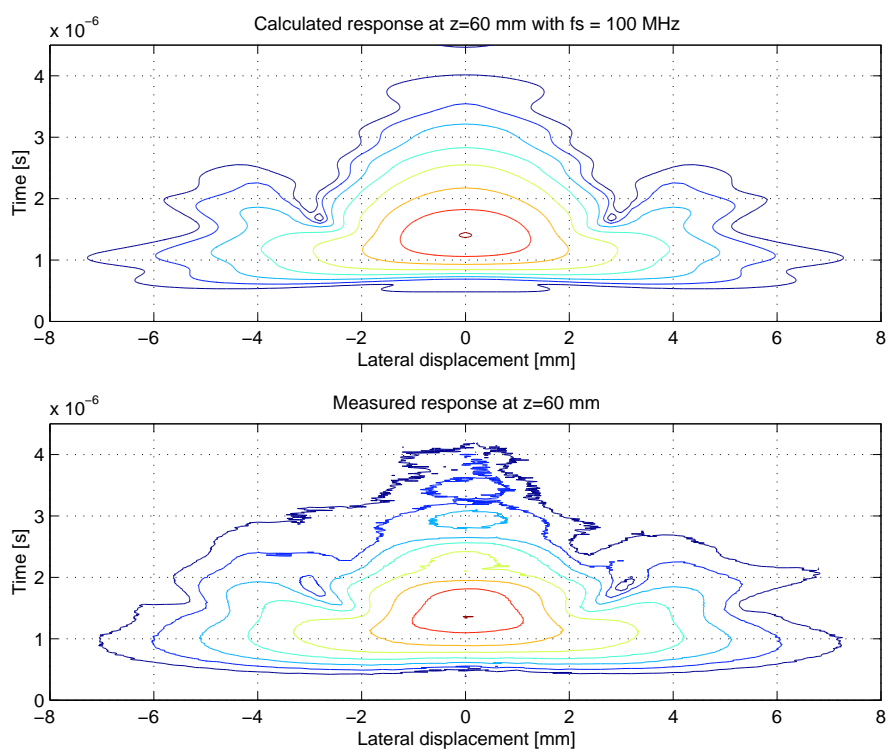

Fig. 14. Point spread function for concave, focused transducer top: simulation, bottom: tank measurement ( $6 \mathrm{~dB}$ between the contour lines). 
signal was recorded as a function of lateral needle position. The slight differences between the graphs can be attributed to noise and measurement inaccuracies.

A quantitative analysis of the simulation errors has been performed. The simulation error is calculated by

$$
e r r=\sqrt{\frac{\sum_{n=0}^{N-1}\left(r_{c}(n)-r_{t}(n)\right)^{2}}{\sum_{n=0}^{N-1} r_{t}^{2}(n)}}
$$

where $r_{t}(n)$ is the reference response calculated at a high sampling frequency and $r_{c}(n)$ is the response to be error evaluated. All error simulations are performed for a 32-element linear-array transducer (element width $0.15 \mathrm{~mm}$, kerf $0.05 \mathrm{~mm}$, height $10 \mathrm{~mm}$, focus $60 \mathrm{~mm}$ from aperture). A pulse-echo simulation using 2000 random scatterers placed in box of 10 by 5 by $5 \mathrm{~mm} 30 \mathrm{~mm}$ from aperture was used.

The result, when using the different mathematical elements, is shown in Fig. 15. It is

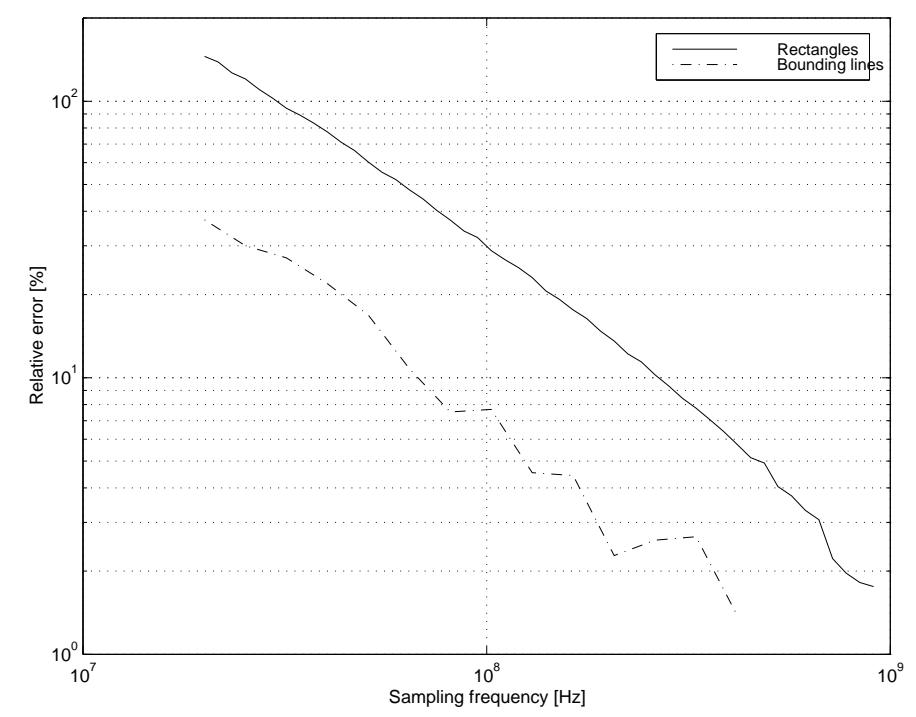

Fig. 15. Accuracy of pulse-echo response for rectangular and bounding lines elements as a function of sampling frequency.

seen that a higher sampling frequency gives a better precision for both the far-field rectangle solution and the bounding line solution. The bounding line solution is better at attaining a high precision at low sampling frequencies due to the exact solution employed and the more advanced integration routine. A factor of roughly 2.5 improvement is seen. The $20 \%$ error for the rectangles might seem high, but it should be remembered that RF signals are compared. An even slight change in the phasing of the response can lead to a very high error. This is especially true at low sampling frequencies. Comparing only the envelope 


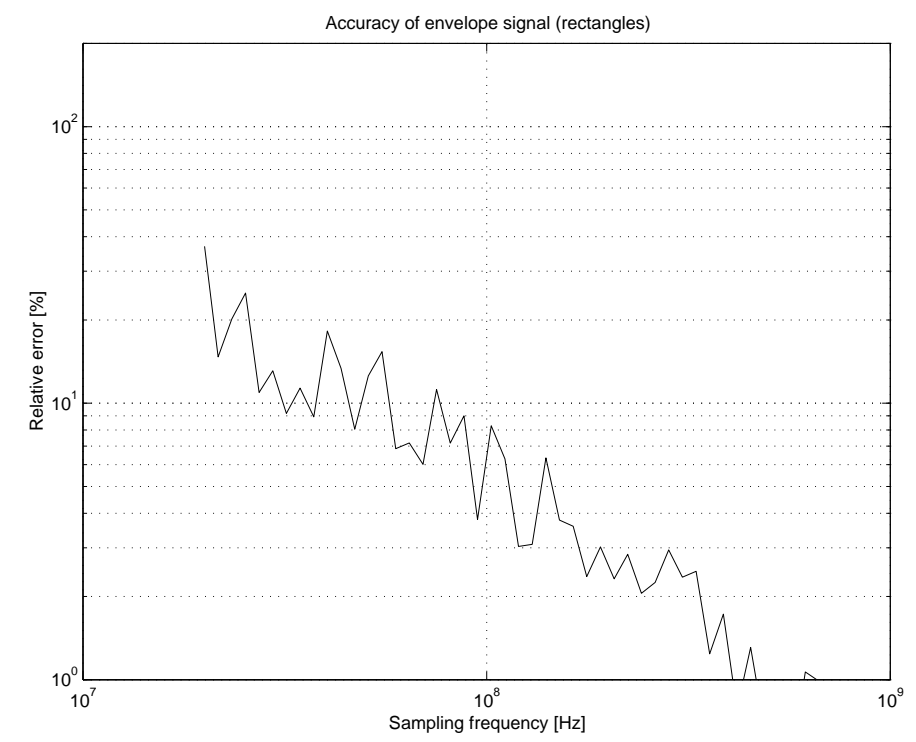

Fig. 16. Accuracy of envelope pulse-echo response for rectangular elements as a function of sampling frequency.

of the responses as in Fig. 16 gives a more favorable error. Here the error is reduced by a factor of $2-3$ compared to the RF response. The envelope is usually also what is presented in ultrasound images.

The relation between number of rectangles, accuracy, and simulation time can be seen in Fig. 17. The top graph show the accuracy as a function of the number of rectangular elements compared to the ideal response. It is seen that the accuracy increases as a function of elements and attains a value of roughly $1 \%$, when using 400 mathematical elements for each physical element. The simulation time per scatterer for both bounding lines and triangles with no subdivision are also shown for comparison in the lower graph. They are a lot slower to evaluate due to the exact solution used and the integration of the responses. But for high precision simulation it can be an advantage to use them, since the storage used for the transducer description is much smaller.

\section{References}

1. G. E. Tupholme. Generation of acoustic pulses by baffled plane pistons. Mathematika, 16:209-224, 1969.

2. P. R. Stepanishen. The time-dependent force and radiation impedance on a piston in a rigid infinite planar baffle. J. Acoust. Soc. Am., 49:841-849, 1971.

3. P. R. Stepanishen. Transient radiation from pistons in an infinite planar baffle. J. Acoust. Soc. Am., 49:1629-1638, 1971.

4. J. A. Jensen. A model for the propagation and scattering of ultrasound in tissue. J. Acoust. Soc. Am., 89:182-191, 1991a.

5. L. E. Kinsler, A. R. Frey, A. B. Coppens, and J. V. Sanders. Fundamentals of Acoustics. John 

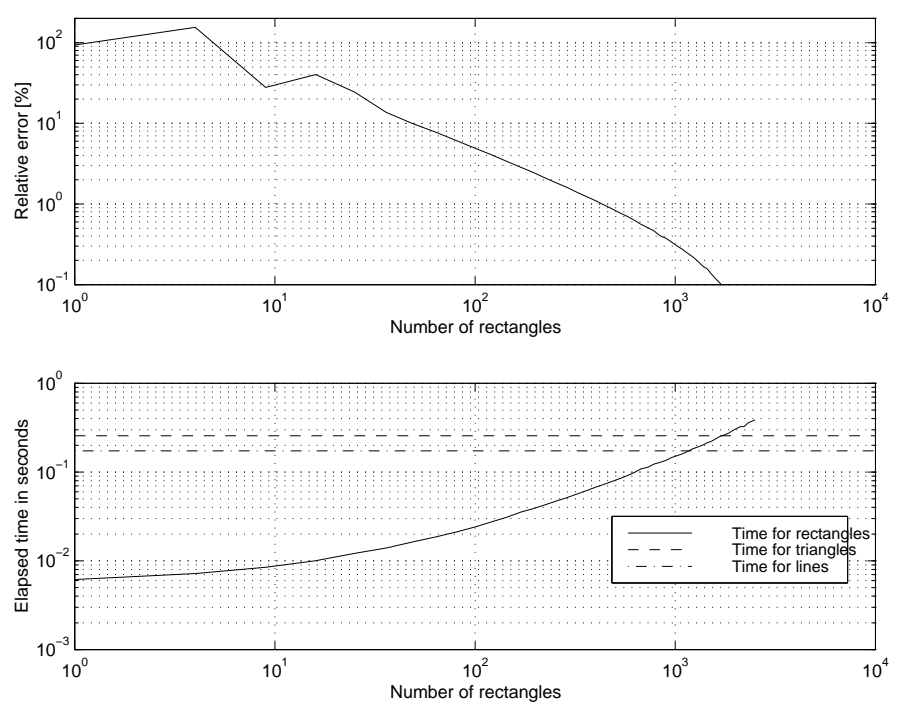

Fig. 17. Accuracy of pulse-echo response when using different number of rectangles for one transducer element and the associated calculation time. The calculation when using triangles or bounding lines is shown as the dashed and dotted lines.

Wiley \& Sons, New York, third edition, 1982.

6. J. A. Jensen and N. B. Svendsen. Calculation of pressure fields from arbitrarily shaped, apodized, and excited ultrasound transducers. IEEE Trans. Ultrason., Ferroelec., Freq. Contr., 39:262-267, 1992.

7. J. A. Jensen. Ultrasound fields from triangular apertures. J. Acoust. Soc. Am., 100(4):2049-2056, 1996a.

8. W. H. Press, B. P. Flannery, S. A. Teukolsky, and W. T. Vetterling. Numerical receipes in C. The art of scientific computing. Cambridge University Press, Cambridge, 1988.

9. J. A. Jensen. A new calculation procedure for spatial impulse responses in ultrasound. J. Acoust. Soc. Am., pages 3266-3274, 1999. 